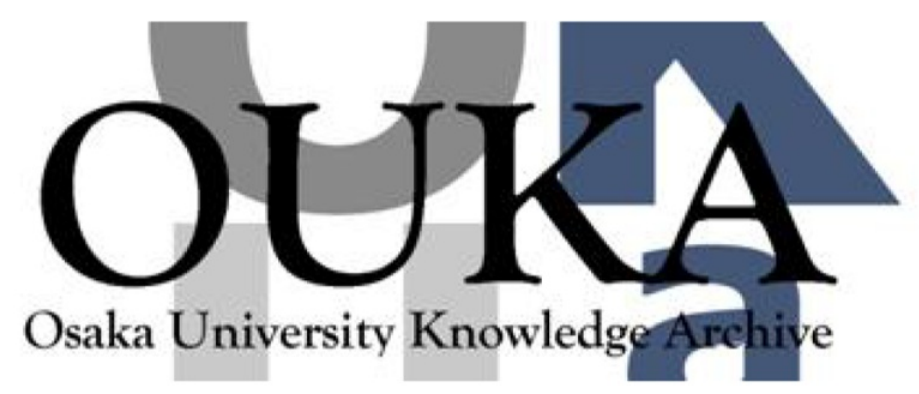

\begin{tabular}{|c|l|}
\hline Title & $\begin{array}{l}\text { Stiffened ultrathin Pt films confirmed by } \\
\text { acoustic-phonon resonances }\end{array}$ \\
\hline Author(s) & Ogi, H.; Fujii, M.; Nakamura, N. et al. \\
\hline Citation & $\begin{array}{l}\text { Physical Review Letters. 98(19) p. 195503-1- } \\
\text { p. 195503-4 }\end{array}$ \\
\hline Issue Date & $2007-05-09$ \\
\hline oaire:version & VoR \\
\hline URL & https://hdl. handle. net/11094/84182 \\
\hline rights & Copyright 2007 by the American Physical Society \\
\hline Note & \\
\hline
\end{tabular}

Osaka University Knowledge Archive : OUKA

https://ir. Library. osaka-u. ac. jp/

Osaka University 


\title{
Stiffened Ultrathin Pt Films Confirmed by Acoustic-Phonon Resonances
}

\author{
H. Ogi, M. Fujii, N. Nakamura, T. Yasui, and M. Hirao \\ Graduate School of Engineering Science, Osaka University, Toyonaka, Osaka 560-8531, Japan
}

(Received 13 December 2006; published 9 May 2007)

\begin{abstract}
Resonances of coherent acoustic phonons were excited and detected by femtosecond light pulses for determining the normal elastic constant of ultrathin platinum films. The elastic constant increases with the decrease of the film thickness, exceeds the bulk value at the thickness near $5 \mathrm{~nm}$, and significantly increases at low temperatures. It shows a correlation with the normal lattice distance. Thus, this Letter provides evidence of the stiffness enhancement in ultrathin films caused by lattice anharmonicity.
\end{abstract}

DOI: $10.1103 /$ PhysRevLett.98.195503

PACS numbers: 62.20.Dc, 68.35.Iv, 78.47.+p

The elastic constants of materials normally represent the proportional coefficients for the linear relationship between stress and strain. However, when the elastic strain becomes larger, the stress-strain relationship departs from linearity because of lattice anharmonicity, and the elastic constants vary from those near the equilibrium volume. The observed changes of the elastic constants are fairly small in usual bulk metals, of the order of $0.1 \%$ or less, even at their failure, because dislocation's slip and multiplication counteract the increase of strain energy. The elastic strain is, however, expected to remain larger in thin films because of much less mobility of dislocations and large intrinsic stress. The evidence of anomalous elastic properties in thin films has been thus an important issue in condensed matter physics.

Superlattice thin films can involve very large elastic strains in individual layers to make coherent bonds at the interfaces, which would alter the elastic constants. The supermodulus effect has been reported in $\mathrm{Au} / \mathrm{Ni}$ and $\mathrm{Cu} / \mathrm{Pd}$ multilayers [1], where the films' elastic constants were significantly larger than those predicted by the bulk elastic constants of the components. Motivated by the discovery of the elastic anomaly, many researchers followed, but most reported negative results for the supermodulus effect [2]. As shown through the perpendicular magnetic anisotropy, a large in-plane strain $\left(\sim 10^{-2}\right)$, should occur at the interfaces in some superlattice systems $(\mathrm{Co} / \mathrm{Pt}$, for example [3] ), but the sign of the strain turns out to be opposite across the interfaces. The periodic compression or tension strains in multilayers cancel the changes of the elastic constants in individual layers, making it difficult to observe the elastic anomaly. Therefore, a monolayer thin film can be an ideal model for the study of the elastic anomaly.

The curvature measurements [4-6] indicated that the inplane internal stress in a deposited thin film experiences compression-tension-compression behavior as the deposition procedure progresses. An earlier theory estimated the maximum tensile stress larger than $1 \mathrm{GPa}$ for a standard metallic material [4]. Floro et al. [6] indicated that the tensile stress in a 2-nm thick Ti film reached 1.8 GPa. Thus, a large in-plane tensile stress could occur at very thin films, and the measurement of an ultrathin-film elastic constant will allow us to observe the effect of lattice anharmonicity. For the evidence of elastic anomaly, however, we must show a stiffened film, not a softened one, because the macroscopic elastic constants are easily lowered by defects [7], incohesive grain bonds [8], and involvement of noncrystallized particles: thin films are generally compliant compared with the bulk materials. For example, the lowered elastic constant was observed in multilayers and it was attributed to interface strains [9], but it is difficult to separate the strain contribution from others. Therefore, the out-of-plane elastic constant of an ultrathin film can be the important target, because the film shrinks in the thickness direction when the in-plane tension occurs. To our knowledge, no study succeeded in the observation of the enhancement of the elastic constant in an ultrathin film based on a reliable measurement.

We here develop a femtosecond pulse-laser method for accurately determining the out-of-plane elastic constant of ultrathin films. Thomsen et al. [10] achieved excitation and detection of high-frequency phonon pulses by ultrafast pulse-laser irradiation. The light pulse (pump pulse) causes acoustic phonons through thermal expansion [10] and diffusion of electrons and their interactions with lattices [11] in metallic materials. The coherent phonon component propagates in the thickness direction as an acoustic plane wave and repeats reflections between the film and the substrate surfaces, which can be detected by the timedelayed light pulse (probe pulse) through the photoelastic effect. Normally longitudinal waves were detected, but shear waves were generated as well from materials showing anisotropic coefficients of thermal expansion [12].

For the evaluation of the elastic properties, the pulseecho signals of the long-distance-propagated phonons have been measured $[9,13,14]$, which required a sufficiently large thickness $(\sim 100 \mathrm{~nm})$ to separately detect the echo signals. However, use of an ultrashort light pulse ( $\sim 100 \mathrm{fs})$ is capable of accelerating phonon vibrations of frequencies up to $\sim 10 \mathrm{THz}$. Some of them remain coherently and resonate within the ultrathin film, causing oscillations in the reflectivity response. Such reverberation signals were 
previously indicated $[14,15]$, but were not used for determining the elastic constants. Here, we clearly identify them for the ultrathin-film elastic constant.

We note platinum because it shows very large biaxial Poisson's ratio on (111) plane as shown in Table I. When an fcc material is deposited on a substrate, the closed-packed plane (111) is predominantly parallel to the film surface, and it shows transverse elastic isotropy with five independent $C_{i j}[7,8]$. The intrinsic stress and the thermalexpansion effect cause the equibiaxial stress field in the film. We define the ratio of the out-of-plane strain $S_{3}$ to the in-plane biaxial strain $S_{1}\left(=S_{2}\right)$ as biaxial Poisson's ratio $\nu_{\mathrm{bi}}=-S_{3} / S_{1}=2 C_{13} / C_{33}$, which indicates how easily the films deform in the out-of-plane direction responding to the in-plane strain. Values in Table I were calculated by averaging the (111) elastic-constant matrix about the $\langle 111\rangle$ direction using the Hill-averaging method [8].

We deposited the Pt thin films on (100) Si substrates by the magnetron-sputtering method with the deposition rate of $1.8 \AA / \mathrm{s}$. The substrates were cleaned in the piranha solution $\left(98 \% \mathrm{H}_{2} \mathrm{SO}_{4}: 33 \% \mathrm{H}_{2} \mathrm{O}_{2}=4: 1\right)$ before deposition. The base pressure was $5 \times 10^{-6} \mathrm{~Pa}$. The film thickness was determined by the $\mathrm{x}$-ray total reflectivity measurement [16]. It can be inversely determined by fitting the theoretically calculated reflectivity coefficient to the measurement. The high-angle x-ray diffraction (XRD) measurement showed very strong (111) texture, and the diffraction peaks from other planes were negligible; the second highest diffraction peak was from (311) planes, whose height was a part of 1000 of that of (111) peak. Thus, we consider the complete (111) textured film in this Letter. The XRD measurement determined $S_{3}$ through (111) plane distance. As a reference, we determined the plane distance of a bulk (unstrained) Pt plate of $1 \mathrm{~mm}$ thick by the same measurement condition and evaluated $S_{3}$ of films from the relative changes.

A mode-locked Ti:sapphire pulsed laser (100 fs duration, $80 \mathrm{MHz}$ repetition rate, and $800 \mathrm{~nm}$ wavelength) was focused onto the film surface as the pump pulse with a spot diameter of $50 \mu \mathrm{m}$ and power of $20 \mathrm{~mW}$. The frequencydoubled probe pulse $(400 \mathrm{~nm})$ was perpendicularly focused on the surface with a time delay and the reflected beam entered into a balanced photo detector together with a reference beam to detect the relative change of the surface reflectivity. The delay time was controlled by changing the light path of the pump pulse. Figure 1 shows the typical responses of the time-resolved reflectivity variation $\Delta R / R$ and the corresponding Fourier spectra. We sub- tracted the slowly decreasing temperature-affected backgrounds. When the film is as thick as $\sim 70 \mathrm{~nm}$, the pulse echoes were separately detected [Fig. 1(a)], which gave the round-trip time and then the out-of-plane longitudinalwave modulus. For ultrathin films, we observe two oscillations [Fig. 1(b)]. The first oscillation appears just after the excitation and quickly decays. The second oscillation follows with lower attenuation. The resonant spectrum identified the second oscillation as the one caused by the interference between the light reflected at the substrate surface and the light diffracted by the acoustic wave propagating into the substrate [17]. This oscillation frequency is given by $2 n v / \lambda$ for the normal incident light, where $n, v$, and $\lambda$ denote the refractive index of the substrate, the sound velocity in the substrate, and the wavelength of the light. Taking $n=5.57$ at $\lambda=400 \mathrm{~nm}$ [18] and $v=8437 \mathrm{~m} / \mathrm{s}$ for $\mathrm{Si}$, we predict the oscillation frequency of $235 \mathrm{GHz}$, which agrees well with the peak observed in the spectra [Figs. 1(c) and 1(d)]. Therefore, the other peaks are considered as the local resonances of acoustic phonons given by $f_{m}=m \sqrt{C_{33} / \rho} /(2 d) . C_{33}$ and $\rho$ are the out-of-plane longitudinal-wave modulus and the mass density of the film, and $d$ is the film thickness. $m$ denotes the mode index number. As shown in Fig. 1(c), the acoustic-phonon resonances up to $m=3$ were observable. Using the bulk mass density and the fundamental resonance frequency $\left(f_{1}\right)$, we determined the modulus $C_{33}$. For thicker films $(d>$ $40 \mathrm{~nm}$ ), we determined it by measuring the round-trip time from the pulse-echo signals.

Figure 2 shows thickness dependences of $C_{33}$, internal friction $Q^{-1}$, and the (111)-plane distance. As the film thickness decreases, the modulus first decreases slowly, and then increases rapidly for $d<\sim 20 \mathrm{~nm}$, where $S_{3}$ changes from positive (tension) to negative (compression). $C_{33}$ exceeds the ideal bulk value $\left(C_{\langle 111\rangle}\right)$ at $d \sim 5 \mathrm{~nm}$. This increase is significant because the negative factors (nanocracks and incohesive bonds) to deteriorate the stiffness must coexist in ultrathin films. One may attribute this stiffness enhancement to the Pt silicides which would be formed near the interface, but this effect will not contribute to the stiffness increase because typical $\mathrm{Pt}$ silicides $\left(\alpha-\mathrm{Pt}_{2} \mathrm{Si}\right.$ and $\mathrm{PtSi}$ ) show lower longitudinal-wave moduli than that of Pt [19]. Thus, the modulus enhancement has been confirmed in ultrathin Pt films. We also determined the apparent internal friction from the peak width for the resonance measurements and from attenuation of the pulse-echo signals for the pulse-echo measurements. The major part of internal friction arises from the energy loss

TABLE I. Biaxial Poisson's ratio $\nu_{\mathrm{bi}}$ and the sensitivity of the modulus $C_{\langle 111\rangle}$ to the biaxial inplane strain $S_{1}$ of (111) textured fcc materials.

\begin{tabular}{lcccccccc}
\hline \hline & $\mathrm{Pt}$ & $\mathrm{Ag}$ & $\mathrm{Al}$ & $\mathrm{Au}$ & $\mathrm{Cu}$ & $\mathrm{Ni}$ & $\mathrm{Pb}$ & $\mathrm{Pd}$ \\
\hline$\nu_{\mathrm{bi}}$ & 1.20 & 0.88 & 1.00 & 1.27 & 0.73 & 0.59 & 1.07 & 1.01 \\
$\left(d C_{\langle 111\rangle} / d S_{1}\right) / C_{\langle 111\rangle}$ & 6.65 & 6.09 & 4.14 & 4.53 & 2.49 & 1.92 & 6.89 & 4.62 \\
\hline \hline
\end{tabular}



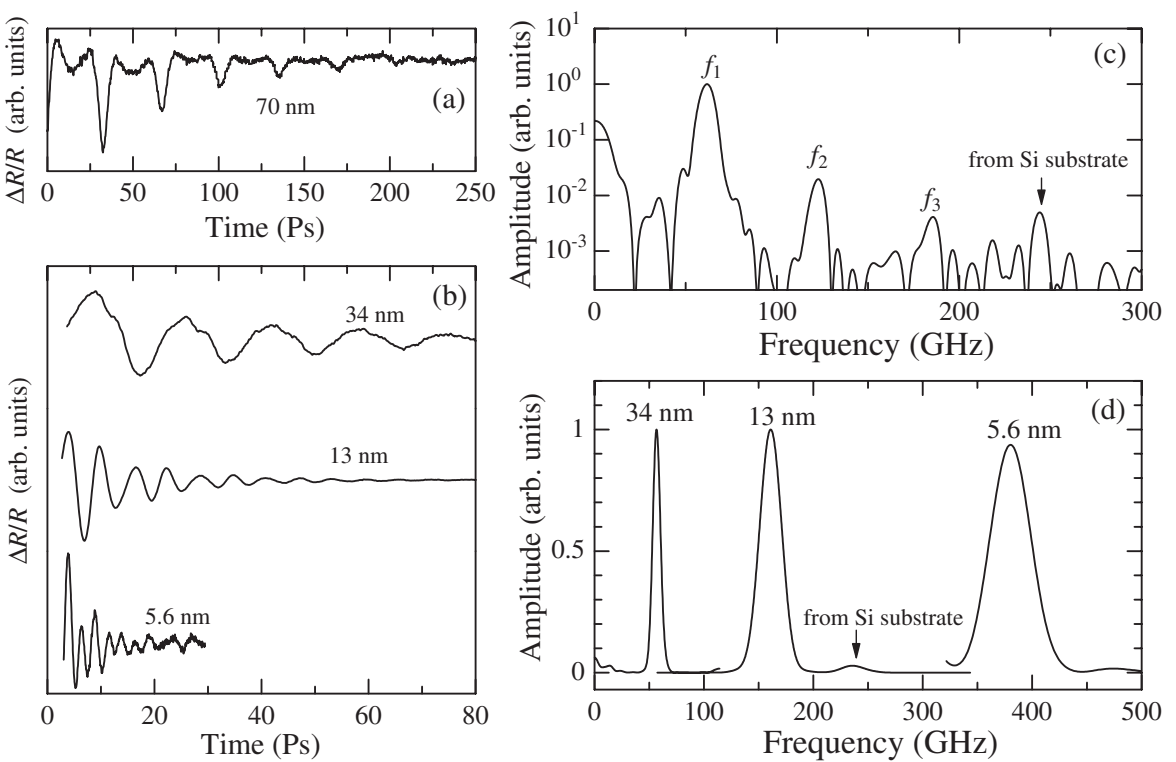

FIG. 1. (a) Pulse-echo signals of the reflectivity variation observed in a 70$\mathrm{nm}$ thick Pt film. (b) Oscillations of the reflectivity in ultrathin Pt films. For the 5.6-nm thick film, the oscillation due to the diffracted light from the Si substrate was digitally filtered. (c) The Fourier spectrum for a 34-nm thick Pt film, showing the acoustic-phonon resonances up to the 3rd overtones. (d) The Fourier spectra for the time-resolved reflectivity responses for various thick films. into the substrate. We estimate this using the acoustic impedances and obtain $Q^{-1} \simeq 0.15$, being comparable with the observation. Internal friction is lowered when the film thickness is smaller. Because internal friction would have increased when many defects existed, the lower internal friction indicates dense and less-defective microstructures. We observed the microstructures by a field-emission scanning electron microscopy and found no visible defects and confirmed that they were continuous, not island microstructures (see the inset at $d=8.5 \mathrm{~nm}$ in Fig. 2).

The thickness dependence of the modulus correlates well with that of the out-of-plane strain, and we intend to calculate the change of the elastic constant by the latticeanharmonicity theory. Thurston and Brugger [20] studied the equations of small-amplitude waves in a homogeneously deformed material and reached the propagation conditions $\rho_{0} W^{2} U_{i}=w_{i j} U_{j}$, where $\rho_{0}, W, U_{i}$ denote the mass density at the unstrained state, the natural sound velocity, and the natural displacement. Thus, $\rho_{0} W^{2}$ corresponds to the governing elastic constant, and they are eigenvalues of the second-rank tensor $w_{i j}$ given by

$$
w_{i j}=N_{k} N_{l}\left[\delta_{i j} \tilde{t}_{k l}+\left(\delta_{j m}+2 \eta_{j m}\right) \tilde{c}_{i k m l}\right] .
$$

Here, $\quad \tilde{t}_{i j}=c_{i j k l}^{0} \eta_{k l}+\frac{1}{2} C_{i j k l m n} \eta_{k l} \eta_{m n} \quad$ and $\quad \tilde{c}_{i j k l}=$ $c_{i j k l}^{0}+C_{i j k l m n} \eta_{m n} ; c_{i j k l}^{0}$ and $C_{i j k l m n}$ are the second-order elastic constants at unstrained state and the third-order elastic constants. $N_{i}$ is the eigenvector, which is along the propagation direction. $\eta_{i j}$ denote the homogeneous Lagrangian strains. Thus, using the reported values of $c_{i j k l}^{0}$ and $C_{i j k l m n}$ [21], we calculated the change of the elastic constants in the presence of the biaxial strain field: first, the biaxial field in the (111) plane coordinate system was converted into that in the usual cubic coordinate system to obtain $\eta_{i j}$. Then, the longitudinal-wave velocity along the $\langle 111\rangle$ direction was determined by solving the eigenvalue problem for $w_{i j}$. The results are shown in Table I, demonstrating the high sensitivity of $C_{\langle 111\rangle}$ of $\mathrm{Pt}$ to the biaxial in-plane strain $S_{1}$, owing to large biaxial

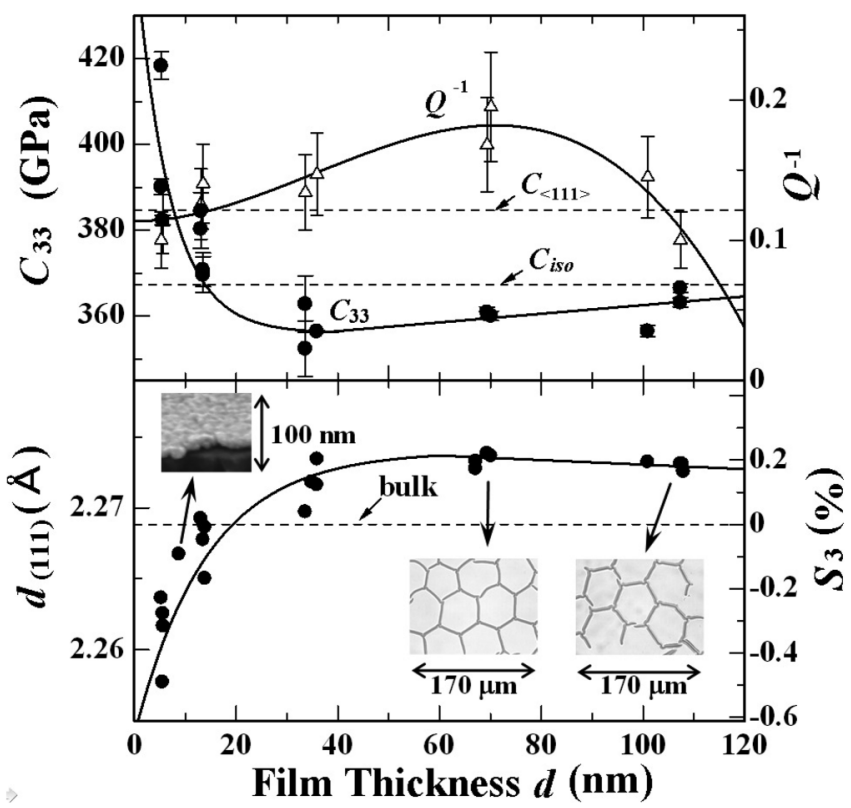

FIG. 2. Thickness dependence of the out-of-plane modulus, internal friction (upper), and the distance between the normal (111) plane (lower) of Pt thin films. The broken line in the lower figure denotes the measurement of an unstrained bulk Pt. $C_{\langle 111\rangle}$ is the longitudinal-wave modulus along the $\langle 111\rangle$ direction of bulk $\mathrm{Pt}$, and $C_{\text {iso }}$ is the modulus of the aggregated isotropic Pt. The inset for the $8.5 \mathrm{~nm}$ thick film confirms a continuous microstructure. Two insets for 70 and $100 \mathrm{~nm}$ thick films show the crashed surfaces, which occurred after 20 days from the deposition; all the measurements had been completed before the inplane buckling occurred. 
Poisson ratio and larger third-order elastic constants in magnitude.

The XRD results in Fig. 2 indicate that $S_{3}=-0.55$ and $0.2 \%$ at $d=5.5$ and $107 \mathrm{~nm}$, respectively, and the calculation predicts the increase and decrease of $C_{33}$ by 9.6 and $3.5 \mathrm{GPa}$ relative to $C_{\langle 111\rangle}$, respectively, which approximately explains the observed modulus changes. The observed changes are, however, larger in magnitudes, indicating that the absolute values of $C_{i j k l m n}$ may be larger than those reported.

We show an obvious evidence of a large compressive inplane stress for thicker films. The films of 70 and $107 \mathrm{~nm}$ thick showed the in-plane buckling to develop beautiful hexagonal ridges after 20 days since the deposition (see insets in Fig. 2). These are caused by the in-plane stress condensation [22,23], and the ridge width $w$ is related with the film thickness and the ridge length $X$ as $w=d^{1 / 3} X^{2 / 3}$ [23]. We calculated $w$ from this equation using $X$ from the optical-microscopy images and compared them with the observed $w$. The calculations yielded $w=3.1$ and $5.4 \mu \mathrm{m}$ for 70 and $107 \mathrm{~nm}$ thick films, respectively, which agree with the observed values of 3.9 and $4.6 \mu \mathrm{m}$. Therefore, the biaxial compressive stress existed in the thicker films.

Furthermore, we measured the change of $C_{33}$ at a low temperature $(77.5 \mathrm{~K})$ : the specimen was attached on a $\mathrm{Cu}$ heat exchanger, which was connected to a liquid-nitrogenfilled stainless-steel chamber located inside a high-vacuum cryostat. The laser beams irradiated the specimen surface via a sapphire window. We repeated the measurement of $C_{33}$ at room temperature and $77.5 \mathrm{~K}$ several times to confirm repeatability of the modulus change. The elastic constants of bulk Pt at low temperatures [24] predict about $2 \%$ increase of $C_{33}$ at $77.5 \mathrm{~K}$, but we observed much larger increases for the ultrathin films. $C_{33}$ increased by 7.8, 4.4, and $2.8 \%$ at $77.5 \mathrm{~K}$ for thicknesses $5.5,13$, and $107 \mathrm{~nm}$, respectively. Therefore, the modulus increase in the ultrathin film is too large to be explained only by the temperature effect. Considering the mismatch of the thermal coefficients, a $220-\mathrm{K}$ decrease of temperature will cause the additional biaxial strain $S_{1}=0.0014$ and $S_{3}=$ -0.0017 in the Pt layer, corresponding to the increase of $C_{33}$ by about $1 \%$ according to the above analysis. Totally, $3 \%$ increase is expected at least. This is an approximate based on the linear dependence of the elastic constants when the strain field is small in magnitude. However, because the energy is increased more steeply by the decrease of the interatomic distance, the large compressive strain will cause much larger increase of the elastic constant when the shrinkage begins from the precompressed state. Therefore, the modulus increase at a low temperature can be more remarkable in the prestiffened films.

We have measured $C_{33}$ of $\mathrm{Ni}, \mathrm{Co}$, and $\mathrm{Cu}$ films as well. They decreased or remained unchanged as the thickness decreased, and their out-of-plane moduli were always smaller than their bulk values. Thus, only Pt showed obvious increase of the out-of-plane modulus, exceeding the bulk value at present.

In conclusion, we observed the evidence of stiffness enhancement in ultrathin Pt films by measuring resonances of acoustic phonons using the femtosecond light pulses. The out-of-plane elastic constant is larger than the bulk value by 5\%-8\% for the films thinner than $10 \mathrm{~nm}$, which was principally explained by the strain dependence of the elastic constant. This enhancement was observed only in Pt thin films so far because of its larger biaxial Poisson's ratio $(>1.2)$ and larger magnitudes of third-order elastic constants.

[1] W. M. C. Yang, T. Tsakalakos, and J. E. Hilliard, J. Appl. Phys. 48, 876 (1977).

[2] J. Mattson et al., J. Appl. Phys. 67, 2873 (1990); B. M. Davis et al., Phys. Rev. B 43, 9304 (1991); H. Huang and F. Spaepen, Acta Mater. 48, 3261 (2000).

[3] T. Kingetsu, J. Appl. Phys. 76, 4267 (1994).

[4] W.D. Nix and B. M. Clemens, J. Mater. Res. 14, 3467 (1999).

[5] R. C. Cammarata, T. M. Trimble, and D. J. Srolovitz, J. Mater. Res. 15, 2468 (2000).

[6] J. A. Floro et al., J. Appl. Phys. 89, 4886 (2001).

[7] H. Ogi et al., J. Appl. Phys. 91, 4857 (2002).

[8] N. Nakamura, H. Ogi, and M. Hirao, Acta Mater. 52, 765 (2004).

[9] B. M. Clemens and G. L. Eesley, Phys. Rev. Lett. 61, 2356 (1988).

[10] C. Thomsen et al., Phys. Rev. B 34, 4129 (1986).

[11] G. Tas and H. J. Maris, Phys. Rev. B 49, 15046 (1994).

[12] O. Matsuda et al., Phys. Rev. Lett. 93, 095501 (2004).

[13] H. T. Grahn et al., Appl. Phys. Lett. 53, 2281 (1988).

[14] B. Perrin et al., Physica (Amsterdam) 219-220B, 681 (1996).

[15] G. A. Antonelli et al., J. Appl. Phys. 91, 3261 (2002).

[16] L. G. Parratt, Phys. Rev. 95, 359 (1954).

[17] A. Devos and R. Côte, Phys. Rev. B 70, 125208 (2004).

[18] E. D. Palik, Handbook of Optical Constants of Solids (Academic, New York, 1998).

[19] O. Beckstein et al., Phys. Rev. B 63, 134112 (2001).

[20] R. N. Thurston and K. Brugger, Phys. Rev. 133, A1604 (1964).

[21] $c_{i j k l}^{0}$ and $C_{i j k l m n}$ of $\mathrm{Ag}, \mathrm{Al}, \mathrm{Au}$, and $\mathrm{Cu}$ are from references given by H. Ledbetter and S. Kim, in Handbook of Elastic Properties of Solids, Liquids, and Gases, edited by M. Levy, H. Bass, and R. Stern (Academic, New York, 2001), Vol. II; $C_{i j k l m n}$ of $\mathrm{Pt}, \mathrm{Ni}, \mathrm{Pb}$, and $\mathrm{Pd}$ are from S. Mathur and P. Gupta, Acustica 31, 114 (1974).

[22] E. M. Kramer and T. A. Witten, Phys. Rev. Lett. 78, 1303 (1997).

[23] T. A. Witten and H. Li, Europhys. Lett. 23, 51 (1993).

[24] R. E. Macfarlane, J. A. Rayne, and C. K. Jones, Phys. Lett. 18, 91 (1965). 